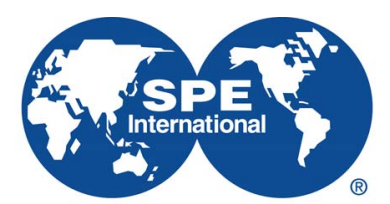

Society of Petroleum Engineers

\title{
SPE-190210-MS
}

\section{Foam Generation by Capillary Snap-Off in Flow Across a Sharp Permeability Transition}

\author{
Swej Y. Shah and Karl-Heinz Wolf, Delft University of Technology; Rashidah M. Pilus, Universiti Teknologi \\ PETRONAS; William R. Rossen, Delft University of Technology \\ Copyright 2018, Society of Petroleum Engineers \\ This paper was prepared for presentation at the SPE Improved Oil Recovery Conference held in Tulsa, Oklahoma, USA, 14-18 April 2018.
}

This paper was selected for presentation by an SPE program committee following review of information contained in an abstract submitted by the author(s). Contents of the paper have not been reviewed by the Society of Petroleum Engineers and are subject to correction by the author(s). The material does not necessarily reflect any position of the Society of Petroleum Engineers, its officers, or members. Electronic reproduction, distribution, or storage of any part of this paper without the written consent of the Society of Petroleum Engineers is prohibited. Permission to reproduce in print is restricted to an abstract of not more than 300 words; illustrations may not be copied. The abstract must contain conspicuous acknowledgment of SPE copyright.

\section{Abstract}

Foam reduces gas mobility and can improve sweep efficiency in an enhanced-oil-recovery process. Previous studies show that foam can be generated in porous media by exceeding a critical velocity or pressure gradient. Such pressure gradients are typically encountered only near a well and therefore, it is uncertain whether foam can propagate far from wells. Theoretical studies show that foam can be generated independent of pressure gradient during flow across an abrupt increase in permeability. In subsurface flow, such sharp permeability changes occur across different length scales. Laminations and cross-laminations, for example, are commonly found small-scale features, whereas unconformities, including layer boundaries and erosional surfaces, are field-scale features that are associated with sharp permeability contrasts across them. In this study, we validate theoretical predictions of foam generation through a variety of experimental evidence. We perform coreflood experiments involving simultaneous injection of gas and surfactant solution at field-like velocities into a model consolidated porous medium made of sintered glass. The core has a well-characterized, sharp permeability transition achieved by sintering glass of different grain sizes. Pressure gradient is measured across several sections of the core to identify foam-generation events and the subsequent propagation of foam. X-ray computerized tomography (CT) provides dynamic images of the coreflood in the form of phase saturations as they develop through the experiment. We investigate the effects of the magnitude of the permeability change and injected gas fractional flow on foam generation and mobilization.

\section{Introduction}

Foams are a distribution of discontinuous gas bubbles in a continuous liquid phase. They can be considered as an example of multiphase 'condensed soft-matter' systems. Foams have numerous applications in the food and chemical industries and in material science. This work focusses on the application of foam to enhanced oil recovery (EOR); more specifically, to capillary-dominated mechanisms of foam generation in porous media. Much of the world's EOR production can be attributed to the injection of gases, especially $\mathrm{CO}_{2}$ and steam. Gas-injection processes, however, are often cursed by unfavourable mobility ratios and differences in fluid densities, which can lead to poor sweep efficiency. In the presence of foam, gas mobility 
is significantly reduced (Bernard \& Holm, 1964; Huh \& Handy, 1989; Rossen, 1996), leading to a more "viscosified" gas that gives better sweep efficiency.

There are three main mechanisms of creation of lamellae (liquid films separating bubbles): namely, lamella division, snap-off and leave-behind (Kovscek \& Radke, 1994; Rossen, 1996). Lamella division, as can be inferred from Figure 1, requires that at least one lamella or lens be initially present. Leavebehind primarily occurs during gas invasion through a liquid-filled medium and is more prevalent during a drainage process.

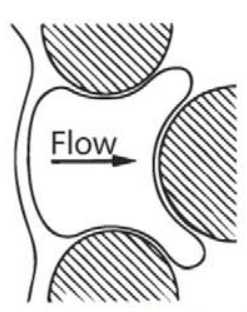

(a)

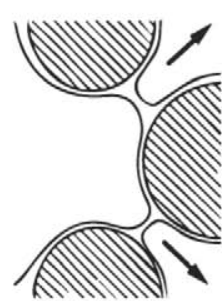

la Division

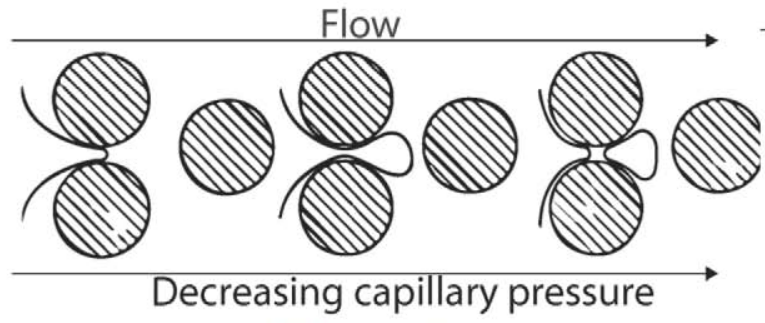

(b) Snap-off

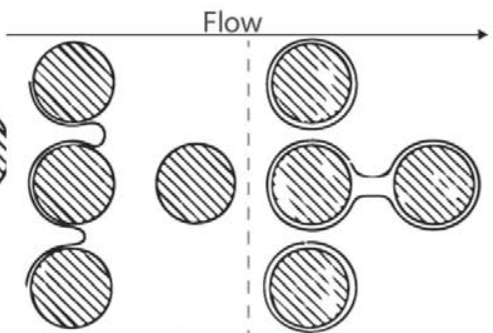

(c) Leave-behind

Figure 1-Mechanisms of lamella creation in porous media (Kovscek \& Radke, 1994)

Foams can be destroyed either by rupturing of the lamellae, for example, through mechanical disturbances or high capillary pressure, or by gas diffusion from smaller to larger bubbles within a pore due to differences in gas pressure. With respect to flow in porous media, foam generation, its propagation and stability, are sensitive to several parameters, including injection rates, local pressure gradient, porosity, permeability, capillary pressure, and oil saturation and composition, among other things. Laboratory experiments show that creation of strong foam in steady gas-liquid flow requires exceeding either a minimum velocity ( $\mathrm{v}^{\mathrm{min}}$ ) or a minimum pressure gradient, denoted as $\nabla P^{\min }$ (Gauglitz et al. 2002; Ransohoff \& Radke 1988; Rossen \& Gauglitz 1990; Tanzil et al. 2002). This criterion depends on the experimental procedure followed, and the threshold may be lower for a drainage process (for example, co-injection of gas and surfactant solution into a liquid-saturated core) owing to assistance by lamellae leave-behind (Rossen \& Gauglitz, 1990). Nevertheless, the minimum-velocity or pressuregradient threshold, as reported in the literature, may only be encountered near a well in a conventional reservoir and is much higher than that encountered away from wells deep inside the formation. This may have unfavourable consequences for foam propagation far from the injection well, as suggested by the experiments of Friedmann et al. (1994) and the modelling of Ashoori et al. (2012), who used the model of Kam (2008), where foam-generation rate is a function of pressure gradient only. Several studies have indicated, however, that foam can be generated independent of pressure gradient, by snap-off, as gas and liquid flow across a sharp increase in permeability (Falls et al. 1988; Hirasaki et al. 2000; Li \& Rossen 2005; Rossen 1999; Tanzil et al. 2002). The extreme case of this phenomenon occurs at the outlet of the core in the form of foam-generation as a consequence of the capillary end-effect. Yortsos and Chang (1990) presented the solution to the mass transport problem for steady gasliquid flow across a permeability jump. Capillary continuity implies an increase in wetting-phase saturation (or reduction in capillary pressure) upstream of the permeability transition, which causes lamella creation by snap-off (van Lingen, 1998).

Sharp changes in permeability are quite common in petroleum reservoirs. Structural features that offer this sort of heterogeneity can exist across a large range of length scales. For example, in laminated and crosslaminated sandstones, the size of each unit could typically range from 1-1000 cm (Reineck \& Singh, 1980). Unconformities such as layer boundaries can extend from a few metres to several hundred metres in length. While vertical anisotropy is more ubiquitous, laterally occurring permeability changes are also important for foam-generation sites because of the driving force of pressure gradient in the horizontal direction. Crosslaminations offer laterally occurring permeability changes oriented perpendicular or at an incline to the 
direction of fluid flow. Figure 2 shows two of the most-common classifications of crossbed units. As can be seen in the figure, the heterogeneity between consecutive laminae is usually a result of zones with contrasting grain size. Hartkamp-Bakker (1993) measured permeability contrasts in outcrop and reservoir core samples with crossbed laminae and reported contrasts ranging from 1:1 to 27:1 between different units.

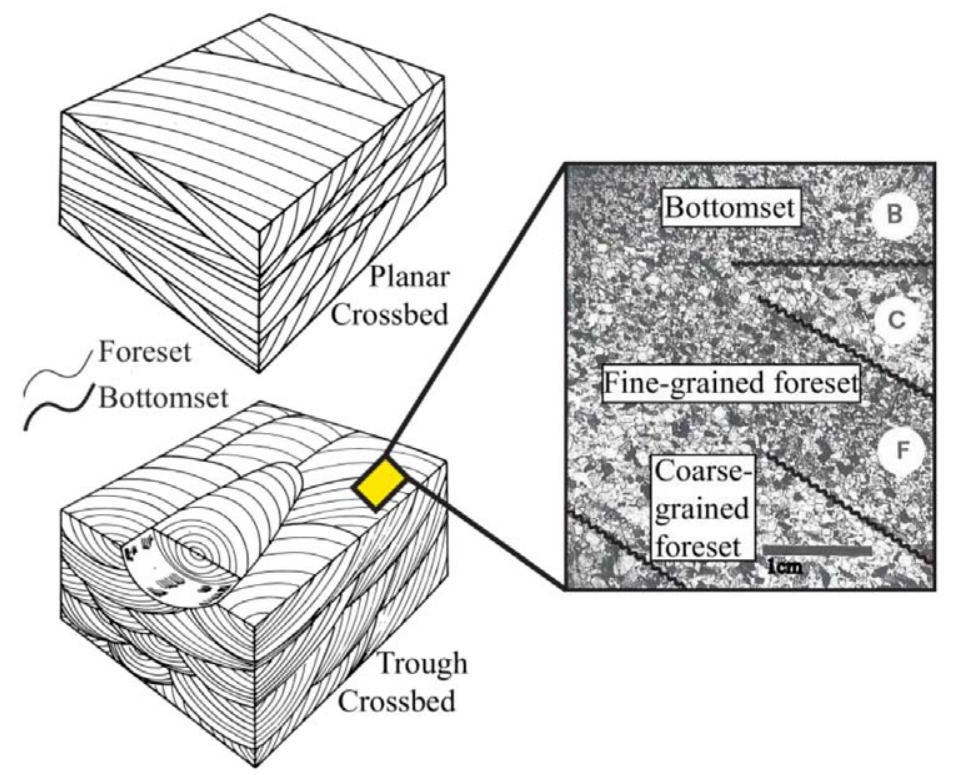

Figure 2-Left - Two common varieties of cross-stratification (Reineck \& Singh, 1980); Right - Changes in grain-size distribution across consecutive laminae as seen in a crossbed thin section from a fluvial outcrop (Hartkamp-Bakker, 1993)

Falls and co-workers (1988) observed foam generation and mobilization experimentally in a beadpack with a permeability contrast of approximately 20:1. They also experimentally measured a critical capillary pressure for snap-off $\left(\mathrm{P}_{\mathrm{c}}^{\mathrm{sn}}\right)$ for the beadpacks used and found it to be approximately half the capillary entry pressure of the medium. For snap-off to occur, enough liquid must accumulate in the low-permeability section to cause the capillary pressure to drop below $\mathrm{P}_{\mathrm{c}}^{\mathrm{sn}}$. Rossen (1999) uses this finding and a pore-network model to illustrate that for "capillary snap-off" to occur at the boundary between two homogeneous regions differing in permeability, the minimum permeability ratio required is 4 . A higher permeability contrast is required for drier flow. Once again, it is important to note that there is no pressure-gradient criterion for the creation of foam through this mechanism, though there may be such a condition for the mobilization of the foam. Tanzil et al. (2002) report visual observations of foam generation and mobilization across such a sharp transition in permeability in their coreflood experiments with sand-packs inside a transparent glass column. They used a permeability ratio of $4.4: 1$ and an injected gas fraction of $67 \%$ for their experiments. Their experimental procedure, however, began with co-injection of gas and surfactant solution into a medium already saturated with surfactant solution, representing a drainage process. As discussed above, other mechanisms of foam generation (such as lamella leave-behind) contribute towards the observed gas mobility reduction during drainage. Contrary to the findings of Tanzil et al. (2002), Li \& Rossen (2005) did not observe foam generation in their sandpack experiments with permeability contrasts of 4.3:1 and 5.1:1. Their experiment started with coinjection of gas and brine into a brine-saturated medium, followed by coinjection of gas and surfactant solution once steady-state has been reached. They suspected that gas bypass along the edges of the pack due to inefficient packing might have been the reason for this observation. They did, however, report foam generation during flow across a much greater permeability contrast of 20:1. The mobilization of this foam was periodic.

In this work, we follow an experimental procedure similar to the work of Li \& Rossen (2005) and use a consolidated porous media to examine the process of foam generation across an abrupt permeability jump. 
The main objective of this work is to validate the theoretical predictions of foam generation (Rossen, 1999) through coreflood experiments that isolate "snap-off" due to a capillary pressure contrast as the only lamellacreation mechanism. We follow field-like superficial velocities to replicate the driving force encountered far from wells in a subsurface reservoir. We measure pressure gradient across several sections of the core to accurately identify the location for the first onset of foam generation and also to observe the propagation of this foam downstream. The experiments are assisted by X-ray computed tomography (CT) to help visualise phase saturations as foam generation and subsequent propagation commences.

This paper is structured as follows. Section 2 describes the experimental set-up, the porous media used and the procedure followed in the experiments reported. Section 3 reports the results of the foamgeneration experiments with measured pressure and CT data obtained through the course of the experiment. The experimental results are analysed and discussed in Section 4, and the paper is concluded in Section 5.

\section{Experimental Design}

\section{Materials and Chemicals}

We perform coreflooding experiments with co-injection of gas and surfactant solution into an artificial porous medium made from sintered borosilicate glass. The cores were acquired from Hilgenberg $\mathrm{GmbH}$, Malsfeld, Germany (www.hilgenberg-gmbh.de). The cores were prepared by sintering crushed, pure borosilicate glass and had a single sharp jump in permeability roughly a third of the way into the length of the core. In other words, roughly $1 / 3$ of the core comprised a homogeneous low-permeability section whereas the rest of the core was made of a homogeneous high-permeability porous medium. The permeability change is achieved by sintering different grain sizes in the same core. The glass grains are angular as can be seen in the picture on the right in Figure 3.

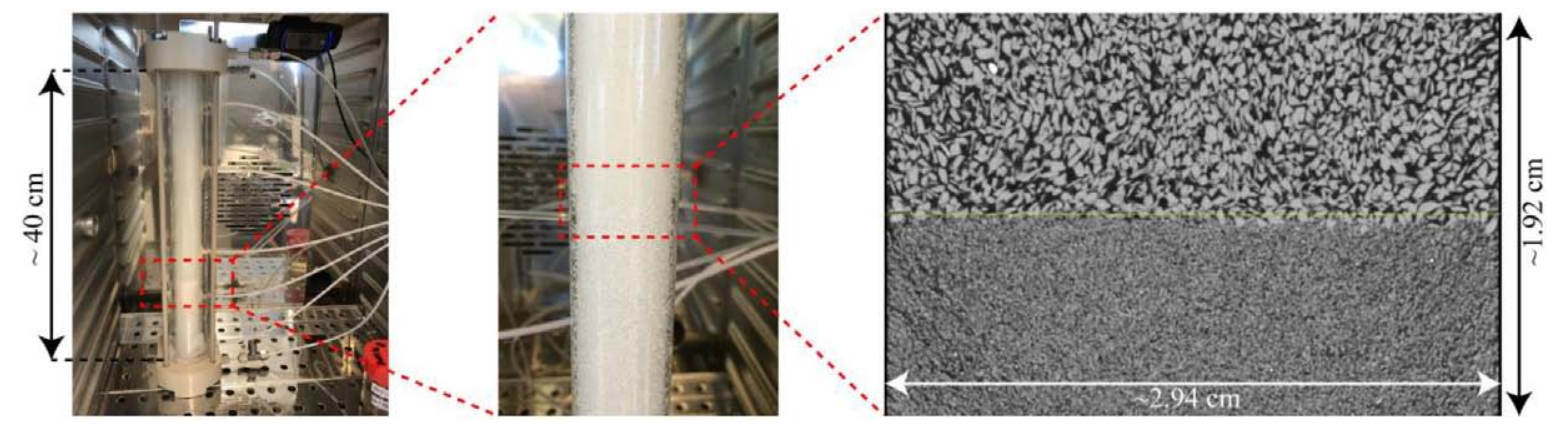

Figure 3-Core holder (left) with sintered-glass core (left and center) and $\mu \mathrm{CT}$ image (with a voxel size of $30 \mu \mathrm{m}$ ) of a vertical crosssection across the permeability change (right).

The cores were prepared in a glass tube with an internal diameter of $3 \pm 0.1 \mathrm{~cm}$ and were cut after the sintering process to roughly $40 \pm 2 \mathrm{~cm}$ in length. The core was enclosed in acrylic glass with polyether-etherketone (PEEK) end-caps. Four different core samples with different permeability ratios were acquired from the manufacturer, the details of which are mentioned in Table 1. The permeability to water is determined from the slope of the straight line formed by a plot of superficial velocity versus the ratio of pressure gradient measured across the two core sections to viscosity (i.e. q/A vs $\nabla P / \mu$ ). The confidence interval for estimating this slope is also reported.

Anionic Alpha olefin sulfonate (AOS) $\mathrm{C}_{14-16}$ with a molecular weight of $315 \mathrm{~g} / \mathrm{mol}$ (Stepan ${ }^{\circledR}$ BIO-Terge AS-40 KSB) was used as a foamer at an active concentration of $0.5 \mathrm{wt} \%(\sim 0.04 \mathrm{M})$. The surfactant solution was prepared using demineralized water also containing $1 \mathrm{wt} . \%(\sim 0.17 \mathrm{M}) \mathrm{NaCl}$. The critical micelle concentration (CMC) of this surfactant in demineralized water with $1 \mathrm{wt} . \% \mathrm{NaCl}$ was measured by Kahrobaei et al. (2017) using the Du Noüy ring method and reported to be 0.008 wt.\%. Therefore, the 
experiments presented in this paper are conducted at roughly 62 times the CMC. The properties of foam films stabilized by this surfactant in the presence of $\mathrm{NaCl}$ as an electrolyte are described by Farajzadeh et al. (2008). Nitrogen $\left(\mathrm{N}_{2}\right)$ with a purity of $99.98 \%$ was used as the gas phase in our experiments.

Table 1-Absolute permeability of individual sections and corresponding permeability contrast in heterogeneous sintered glass core samples used in the experiments

\begin{tabular}{|c|c|c|c|}
\hline Core sample & Pore Size (Specified by Manufacturer) & Permeability & $\left\langle\mathrm{k}^{\text {High }} / \mathrm{k}^{\text {Low }}\right\rangle$ \\
\hline \multirow{2}{*}{1} & $\begin{array}{c}\text { Low perm: } 16-40 \mu \mathrm{m} \\
\text { High Perm: } 40-100 \mu \mathrm{m}\end{array}$ & $\begin{array}{c}\text { Low perm: } 5.4 \pm 0.02 \mathrm{D} \\
\text { High Perm: } 20.7 \pm 0.2 \mathrm{D}\end{array}$ & 3.8 \\
\hline 2 & Low perm: $40-60 \mu \mathrm{m}$ & $\begin{array}{c}\text { Low perm: } 10.9 \pm 0.01 \mathrm{D} \\
\text { High Perm: } 59.3 \pm 0.8 \mathrm{D}\end{array}$ & 5.4 \\
\hline \multirow{2}{*}{3} & High Perm: $100-160 \mu \mathrm{m}$ & Low perm: $3.1 \pm 0.01 \mathrm{D}$ & 13.9 \\
& Low perm: $16-40 \mu \mathrm{m}$ & High Perm: $43.2 \pm 0.2 \mathrm{D}$ & 27.5 \\
\hline
\end{tabular}

\section{Experimental Apparatus}

A schematic flow diagram of the experimental set-up is shown in Figure 4. Pressure gradient is measured every second across several sections of the core. In the low-permeability section, pressure gradient is used to confirm that there is no foam present or being generated as the experiment begins. Across the permeability transition, pressure gradient indicates whether there is any foam being generated at the face of the heterogeneity. In the high-permeability section, pressure drop is used to gain insight into the mobilization of foam generated at the permeability change. We avoid the use of back-pressure regulators and employ atmospheric back-pressure instead, to avoid fluctuations introduced by multiphase flow through the backpressure regulator. Any fluctuation in pressure at the downstream end could travel upstream, causing local fluctuations which would assist in foam generation. Therefore, the outlet of the core is open to atmosphere. The entire apparatus is placed on top of the CT scanner table. The medical CT scanner is inside a temperature-controlled room at $21 \pm 0.4^{\circ} \mathrm{C}$.

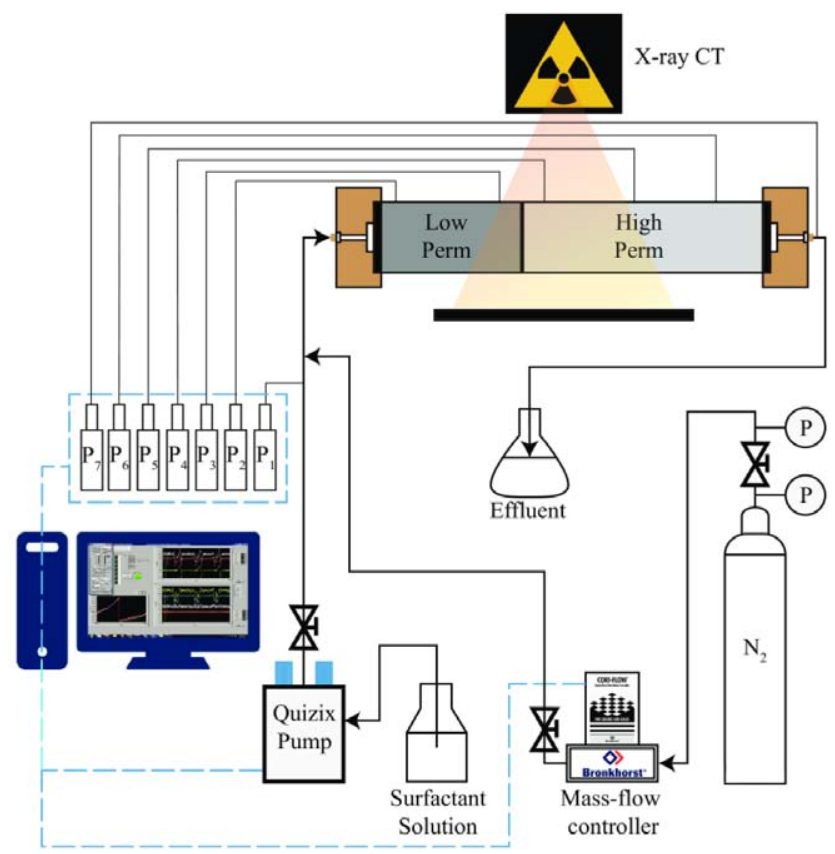

Figure 4-Schematic drawing of the experimental apparatus 


\section{Experimental procedure}

The basic sequence of steps carried out through each coreflooding experiment is as follows:

Permeability measurement. The setup is checked for leaks every time a new core is placed inside the core holder. Following the leak test, $\mathrm{CO}_{2}$ is injected into the core for about 10 pore volumes (PV) to displace air present in the core. The last $2 \mathrm{PV}$ of $\mathrm{CO}_{2}$ are injected under vacuum. While under vacuum, demineralized, degassed water is injected at low flow rates of about $10^{-3} \mathrm{PV} / \mathrm{min}$ into the core to displace the $\mathrm{CO}_{2}$. After water breakthrough, the core is brought to atmospheric pressure by closing the outlet and continuing water injection. Water injection is continued for another PV, following which the permeability of the core is measured by measuring pressure drop across different sections of the core at different flow rates. Next, the core is flushed with $1 \mathrm{wt} . \% \mathrm{NaCl}$ brine and the permeability is calculated again. Measured permeability is used as the first indicator for the presence of any trapped gas in the core, especially when conducting multiple experiments with the same core.

Foam-generation experiment. At the end of the permeability measurement, the core is fully saturated with brine. To start the experiment, brine flow rate is set to the desired value and gas is introduced at the required fractional flow. Once gas-brine injection has reached steady-state, brine injection is replaced by surfactant injection. This procedure ensures that no foam is generated under drainage conditions. During the course of the experiment, we expect to see foam generation in the core. Once the experiment has reached steady state or sufficient data has been acquired, injection is stopped and the core cleaning procedure commences.

Core-cleaning procedure. After each experiment, the core is flushed with approximately $10 \mathrm{PV}$ of $50 \mathrm{wt}$. $\%$ iso-propanol solution to kill the foam. This is followed by around $10 \mathrm{PV}$ of demineralized water injection to remove all the alcohol and remaining surfactant solution from the system. If more experiments are to be performed with the same core, this step is followed by $\mathrm{CO}_{2}$ injection and the permeability-measurement protocol.

\section{Results}

Foam generation was observed in each experiment performed. All the experiments reported in this paper were carried out at a total injection rate $\left(\mathrm{q}_{\mathrm{t}}\right)$ of $0.1 \mathrm{ml} / \mathrm{min}$, on average about $1.4 \mathrm{PV} /$ day for each core, which corresponds to a superficial (Darcy) velocity of $2.36 \mu \mathrm{m} / \mathrm{s}(0.67 \mathrm{ft} / \mathrm{d})$ for each core. This superficial velocity was selected based on observations from a series of tests conducted at different flow rates. The objective of these tests was to select a velocity that was low enough to cause foam generation across the permeability transition only, that registered pressure which was higher than the accuracy of the transducers and would allow each experiment to be completed within a reasonable time frame. At a velocity of 3.34 $\mathrm{ft} / \mathrm{d}(0.5 \mathrm{ml} / \mathrm{min})$, foam generation was observed in the inlet low-permeability section itself; evidently the pressure gradient in the low-permeability section was higher than the minimum pressure gradient required for foam generation. At a velocity of $1.34 \mathrm{ft} / \mathrm{d}(0.2 \mathrm{ml} / \mathrm{min})$, foam generation was observed across the permeability transition and not in the inlet section. However, this total superficial velocity was still higher than that for fields with larger well spacing, as is often the case in offshore developments. At a superficial velocity of $0.67 \mathrm{ft} / \mathrm{d}(0.1 \mathrm{ml} / \mathrm{min})$, we observe foam generation across the permeability jump and no foam in the inlet section, at least at the onset of foam generation across the heterogeneity. At lower flow rates, the experiment would take several weeks to conclude and the fluid velocities through the core would no longer be representative of conventional reservoir flow. Therefore, we selected a total superficial velocity of $0.67 \mathrm{ft} / \mathrm{d}$ for all our subsequent experiments.

\section{Sample 1}

A foam-generation experiment was conducted with sample 1, which has a permeability contrast of 3.8:1, very close to the theoretical prediction of 4 required to cause foam generation by snapoff at $80 \%$ gas 
fractional flow (Rossen, 1999). Figure 5 shows the measured absolute pressure and the corresponding pressure gradient across various sections of the core. The origin of the plot represents the start of surfactant injection into the core, after steady-state has been achieved for the co-injection of gas and brine. Absolute pressure is measured at 7 locations across the length of the core, schematically shown in Figure 4 . In the bottom plot of Figure 5, pressure gradient across 4 sections of the core is plotted: the inlet and outlet sections are ignored for the sake of readability.

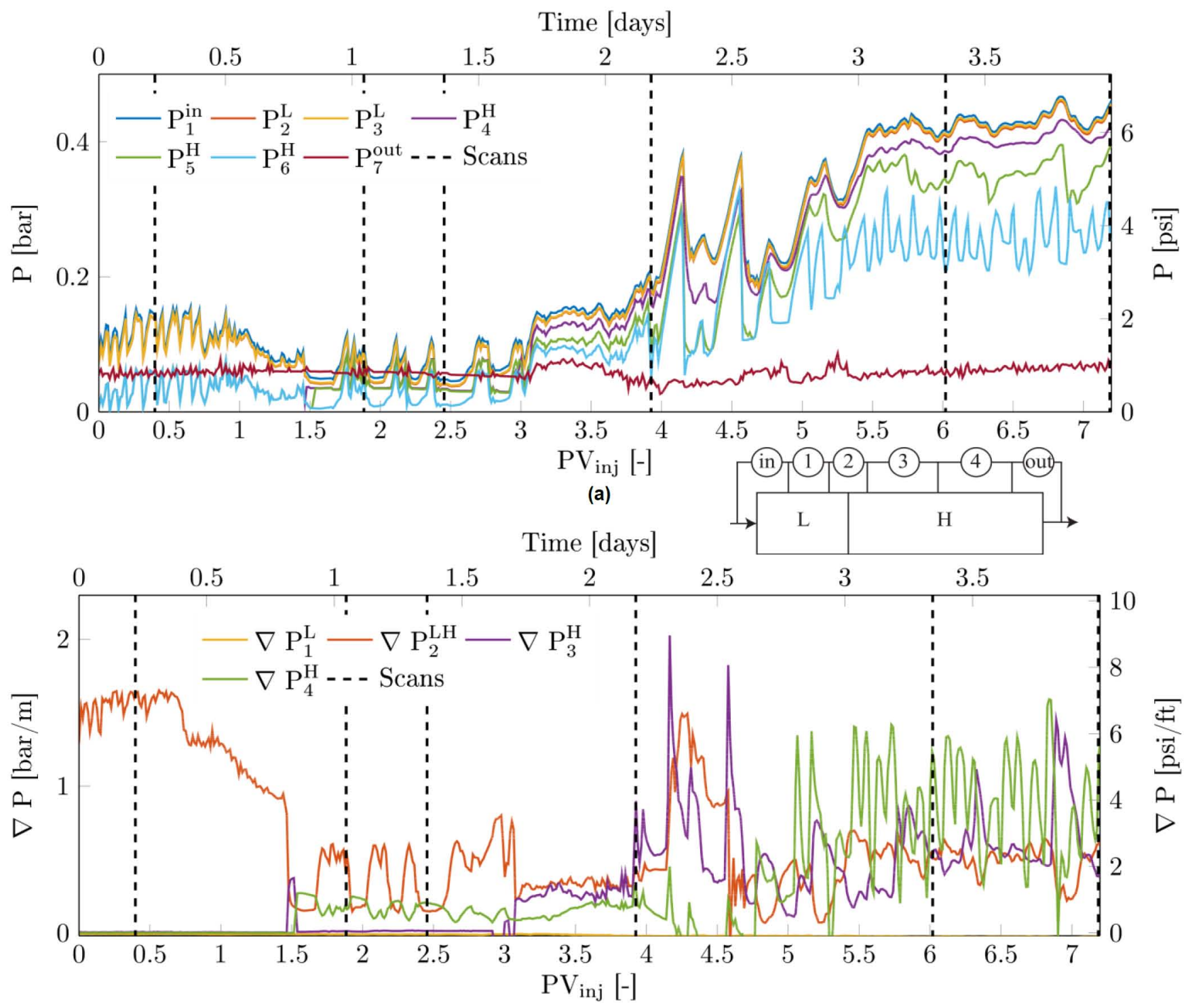

(b)

Figure 5-Absolute pressure (a) and pressure gradient (b) across variations sections of core sample 1 during foam-generation experiment. Dashed lines indicate times at which CT scans were taken to generate saturation maps across the length of the core. Superscript $L$ represents a measurement in the lowpermeability section, whereas superscript $\mathrm{H}$ represents data acquired from the high-permeability zone.

Pressure gradient across the permeability transition rises sharply, soon after gas is introduced into the core at $80 \%$ volume fraction. As co-injection of gas and brine reaches steady-state, $\nabla \mathrm{P}_{2}^{\mathrm{LH}}$ continues to register an abnormally high pressure gradient. We suspect that this may be due to pressure taps across the permeability transition sensing different phases (McCool et al., 1983) which is further aggravated by gas trapping and the "internal" capillary end-effect caused by a sharp difference in capillary pressure between the two zones in this section. After surfactant injection begins, the measured pressure gradient shows a mild and gradual drop 
from about $0.7 \mathrm{PV}$ to $1.5 \mathrm{PV}$. We suspect that this may be because of a diffused surfactant front reducing the interfacial tension and subsequently, the capillary pressure between the two phases at the pressure tap. It could also be due to a gradual build-up in gas saturation near the pressure tap in the high-permeability zone (McCool et al., 1983). In bulk, an increase in surfactant concentration reduces interfacial tension below the CMC. At concentrations beyond the $\mathrm{CMC}$, surfactant concentration has negligible effect on interfacial tension. Therefore, only a small amount of surfactant solution at a concentration much higher than its CMC would be enough to drastically reduce surface tension and capillary pressure.

Pressure gradient $\nabla \mathrm{P}_{1}^{\mathrm{L}}$ across the low-permeability zone is negligible through the course of the experiment, which suggests that there is no significant reduction in mobility or indication of strong foam present or being generated in the inlet section of the core. Dashed lines in Figure 5 indicate times at which CT scans were taken across the entire length of the core. Raw CT data in terms of Hounsfield units (HU) is used to compute porosity and phase saturations as a voxel property for each image stack. Porosity is obtained as (Mees et al., 2003):

$$
\phi=\frac{\mathrm{CT}_{\text {wet }}-\mathrm{CT}_{\text {dry }}}{\mathrm{CT}_{\text {water }}-\mathrm{CT}_{\text {air }}}
$$

where $\mathrm{CT}_{\text {wet }}$ denotes the CT measurement of a liquid-saturated core; we use water as the liquid phase at this stage. $\mathrm{CT}_{\text {dry }}$ is the $\mathrm{CT}$ measurement of a dry core, obtained when the core is not yet saturated with any liquid. $\mathrm{CT}_{\text {water }}$ and $\mathrm{CT}_{\text {air }}$ represent the attenuation values of water and air, in bulk, in Hounsfield units (HU). During the course of the experiment, liquid-phase saturation is computed for each scan as:

$$
\mathrm{S}_{\text {liq }}=\frac{\mathrm{CT}_{\text {exp }}-\mathrm{CT}_{\text {dry }}}{\mathrm{CT}_{\text {liq }}-\mathrm{CT}_{\text {dry }}}
$$

where $\mathrm{CT}_{\text {exp }}$ denotes the $\mathrm{CT}$ measurement taken during the course of the multiphase flow experiment. $\mathrm{CT}_{\text {liq }}$ represents the CT measurement for a core that is fully saturated with the liquid whose saturation is being computed. It is important to note that the accuracy of CT measurements depends on different parameters selected for the X-ray source, such as applied beam voltage, corresponding beam energy and the applied filters for shaping the beam. Figure 6 shows the liquid-phase saturation in the core computed using Equation 2. The saturation is shown across a vertical cross-section through the center of the core during different stages of the experiment. The pixel size in each image slice is $195 \times 195 \mu \mathrm{m}^{2}$ and each slice is $1.5 \mathrm{~mm}$ thick. Liquid-phase saturation values are averaged in each cylindrical slice and plotted across the core length in Figure 7.

When gas and brine are injected into the core, most of the gas overrides to the top and bypasses a large part of the core, as can be seen in the saturation map at the top in Figure 6. Once surfactant is introduced into the core, it appears that foam is being generated in the first section itself, as can be seen in the CT image at 0.4 total pore volumes injected (PVI). This is most likely a very weak foam as it is not persistent and is absent in the images thereafter. Moreover, there is no significant rise in $\nabla \mathrm{P}_{1}^{\mathrm{L}}$ (Figure 5) to demonstrate mobility reduction and the presence of foam, downstream of the inlet. At 2.4 PVI, a modest reduction in gas mobility is observed in the section with the permeability transition (Figure 5), this shows up as a higher gas-phase saturation just at the entrance of the high-permeability zone, clearly discernable in Figure 7. At roughly $3 \mathrm{PVI}$, pressure gradient downstream of the permeability jump, $\nabla \mathrm{P}_{3}^{\mathrm{H}}$ and $\nabla \mathrm{P}_{4}^{\mathrm{H}}$, begin to rise (Figure $5)$ indicating a reduction in gas mobility due to foam propagation. This is verified by the CT response, which shows a foam front propagating through the high-permeability zone at 3.9 PVI. At 6 PVI, CT images show that the foam front has reached the outlet of the pack and there is still no foam in the low-permeability zone. This gives a clear indication of foam generation at the sharp permeability increase and subsequent propagation downstream towards the outlet of the core. 
$\mathrm{S}_{\mathrm{w}} \rightarrow$

0

0.2

0.4

0.6

0.8

1

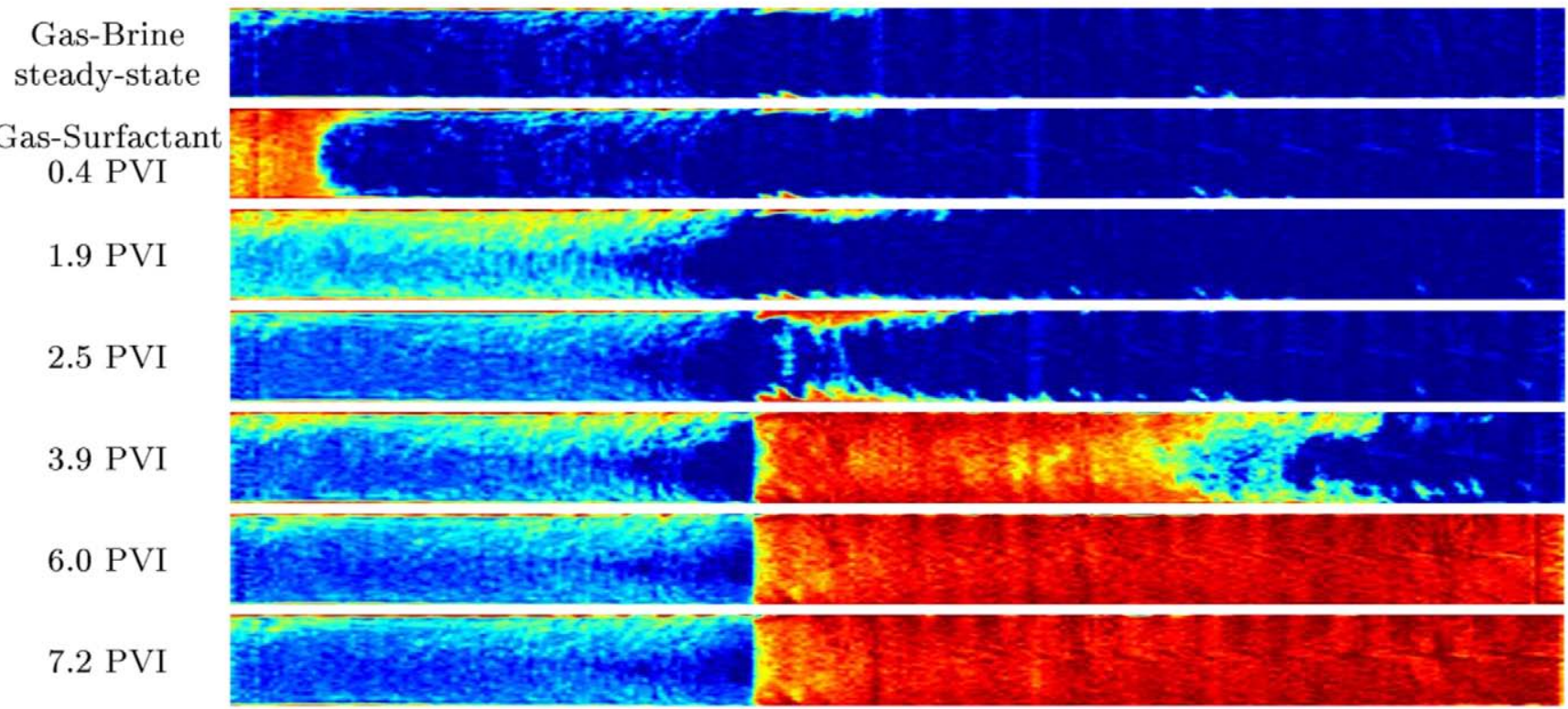

Figure 6-Liquid saturation profile computed using X-ray CT imaging as seen in a vertical cross-section through the center of the core. Color bar represents a liquid saturation range from 0 to 1 . Blue represents a high liquid-phase saturation whereas red represents a high gas saturation, here interpreted as the CT response to the saturation change caused by foam. Top-most saturation map comes from the CT image taken during gas-brine injection and images thereafter were taken after surfactant solution was introduced in the core.

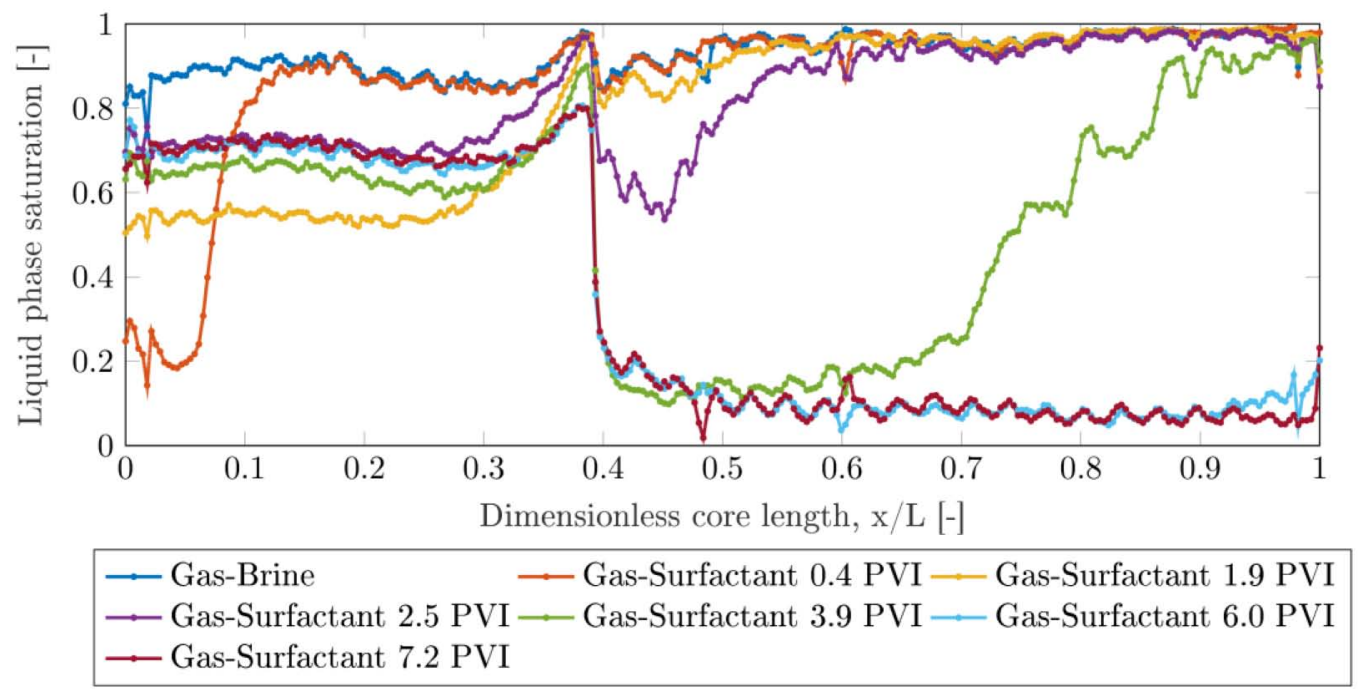

Figure 7-Average liquid-phase-saturation profile in sample 1 at different pore volumes of injection (PVI) through the course of the foam-generation experiments in Figure 6.

\section{Sample 2}

A foam-generation experiment was performed in core sample 2, with a permeability contrast of 5.4:1 between the low- and high-permeability zones. The pressure gradient across different sections of the core developed with time in a manner similar to sample 1, as shown in Figure 8.

Once again, the pressure gradient across the permeability change gradually declines after surfactant is introduced in the core. There is a sharp drop in $\nabla \mathrm{P}_{2}^{\mathrm{LH}}$ and a sharp jump in the downstream pressure gradient $\nabla \mathrm{P}_{3}^{\mathrm{H}}$ at around 2.3 PVI. As shown in Figure 9, CT images at 2.6 PVI show that this coincides with an increase in gas saturation in the high-permeability zone. The images also suggest that the preferred path of 
gas flow is along the edges of the core, which means that the initial onset of foam generation takes place right at the entrance to the high-permeability zone near the walls of the core. This mobility reduction then forces the gas to flow through the center of the core, resulting in foam generation through the whole face of the heterogeneity.

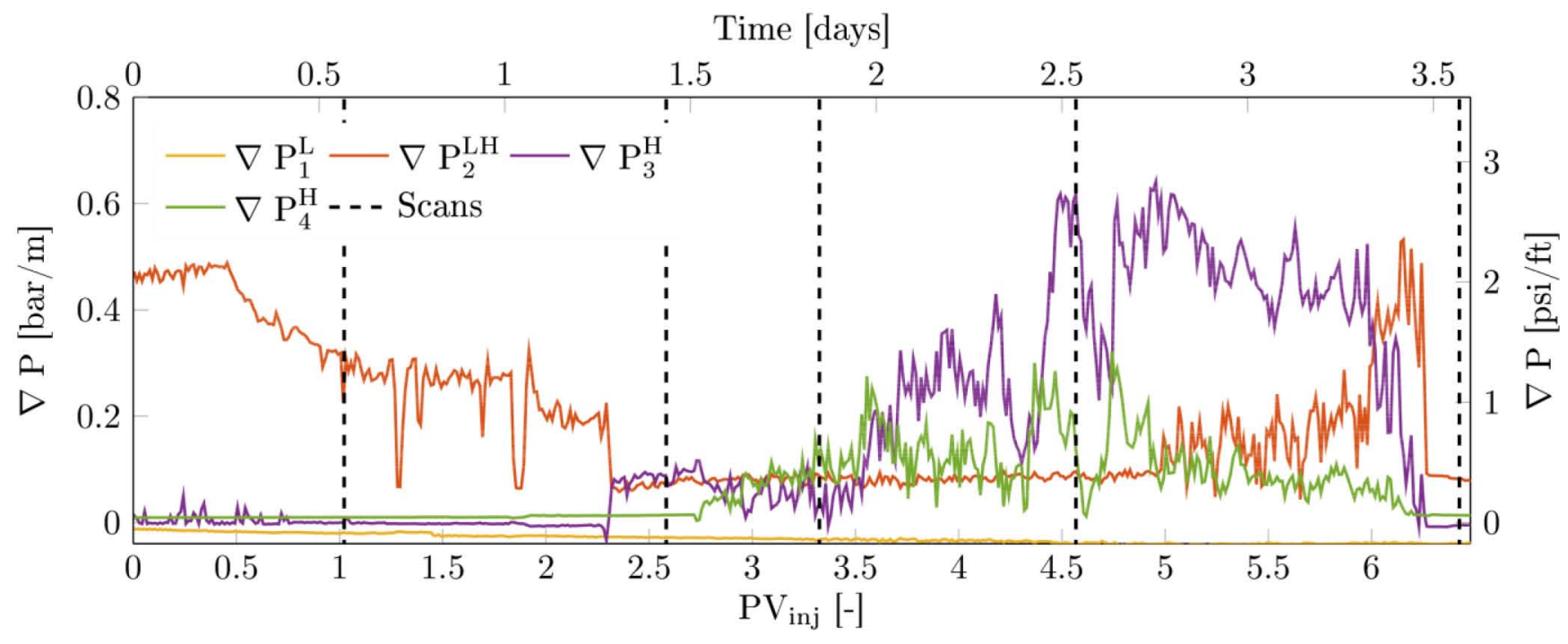

Figure 8-Pressure gradient across variations sections of core sample 2 during foam-generation experiment. Dashed lines indicate times at which CT scans were taken to generate saturation maps across the length of the core. Superscripts $L$ and $H$ denote the lowand high-permeability sections, respectively.

$\mathrm{S}_{\mathrm{w}} \rightarrow$ 0 0.4 0.6 0.8 1

Gas-Brine steady-state

Gas-Surfactant

1.0 PVI

2.6 PVI

3.3 PVI
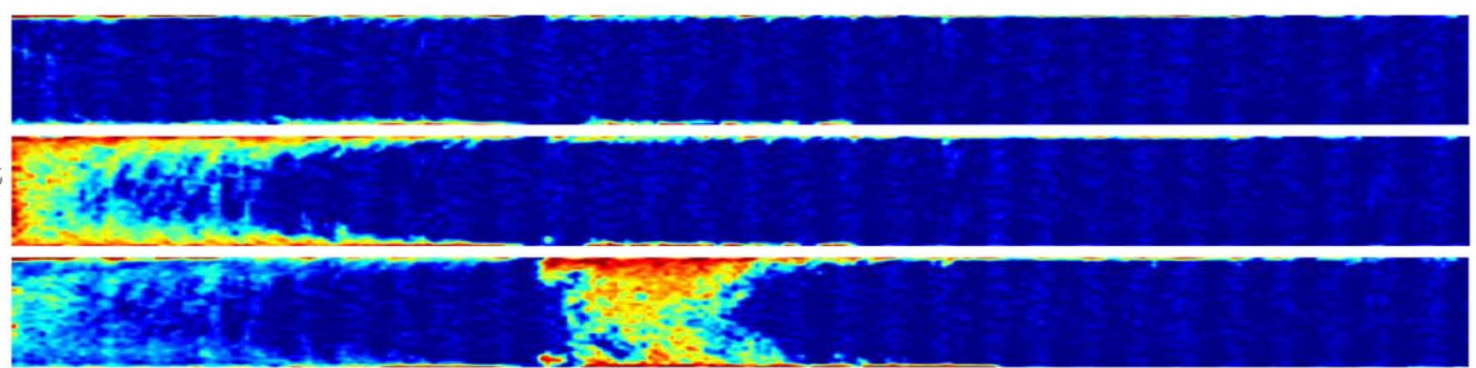

4.6 PVI

6.4 PVI

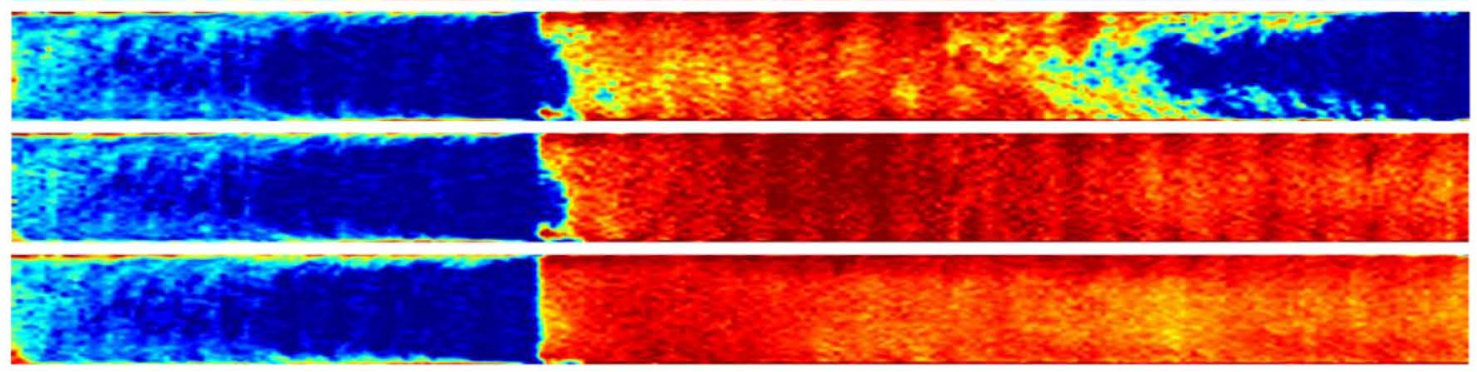

Figure 9-Liquid saturation profile in a vertical cross-section through the center of core sample 2 computed using X-ray CT imaging during the course of a foam-generation experiment. Color bar represents a liquid saturation range from 0 to 1. Blue represents a high liquid-phase saturation whereas red represents a high gas-phase saturation, here interpreted as the CT response to the saturation change caused by foam. Top-most saturation map comes from the CT scan taken during gas-brine injection and images thereafter are from scans taken after surfactant solution was introduced in the core.

Saturation values per cylindrical slice are plotted against the dimensionless core position in Figure 10. The core is almost completely saturated with liquid during co-injection of gas and brine, since most of the gas breaks through from the edges of the core, more from the top than from the bottom. At 2.6 PVI, liquid saturation drops sharply at the entrance of the high-permeability zone, indicating foam generation. 
At 3.3 PVI, the foam front appears to have travelled from a dimensionless position of 0.6 to 0.9 , roughly half the length of the high-permeability zone, into the $4^{\text {th }}$ section of the core. This is also evident from the pressure-gradient profile shown in Figure 8 as $\nabla \mathrm{P}_{4}^{\mathrm{H}}$ begins to rise and exhibit sharp fluctuations, starting at approximately 2.7 PVI. At 4.6 PVI, foam has propagated to the end of the pack and the gas saturation is about $90 \%$ in the high-permeability region. Gas saturation is roughly $20 \%$ in the low-permeability zone and no significant reduction in mobility is witnessed in terms of pressure gradient (Figure 8).

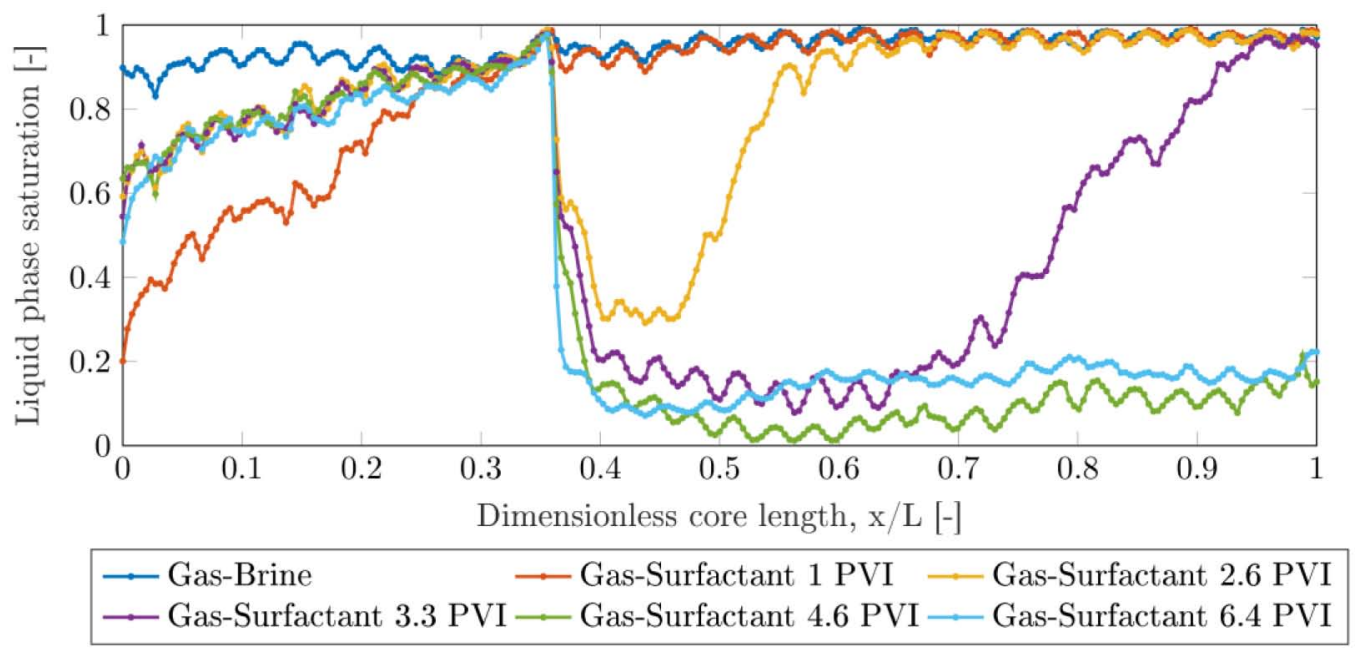

Figure 10-Average liquid-phase saturation profile in core sample 2 plotted at different PVI through the course of the foamgeneration experiment.

\section{Sample 3}

Foam generation was also observed in core sample 3 with a permeability contrast of 13.9:1. As shown in Figure 11, pressure gradient across the permeability transition and further downstream showed distinct periods of rise and sharp drops, unlike the continuous and relatively mild fluctuating pattern observed in Figure 5 and Figure 8. The pressure gradient in the low-permeability section $\left(\nabla \mathrm{P}_{1}^{\mathrm{L}}\right)$ stays low through the course of the experiment, suggesting there is no significant reduction in gas mobility. However, as shown in Figure 12 and Figure 13, the gas saturation is approximately 50\% in the first section. This means that if there is any foam in the low-permeability section, it has little effect on gas mobility and is therefore, weak foam. Evidently, even this weak foam transforms into strong foam at the permeability transition.

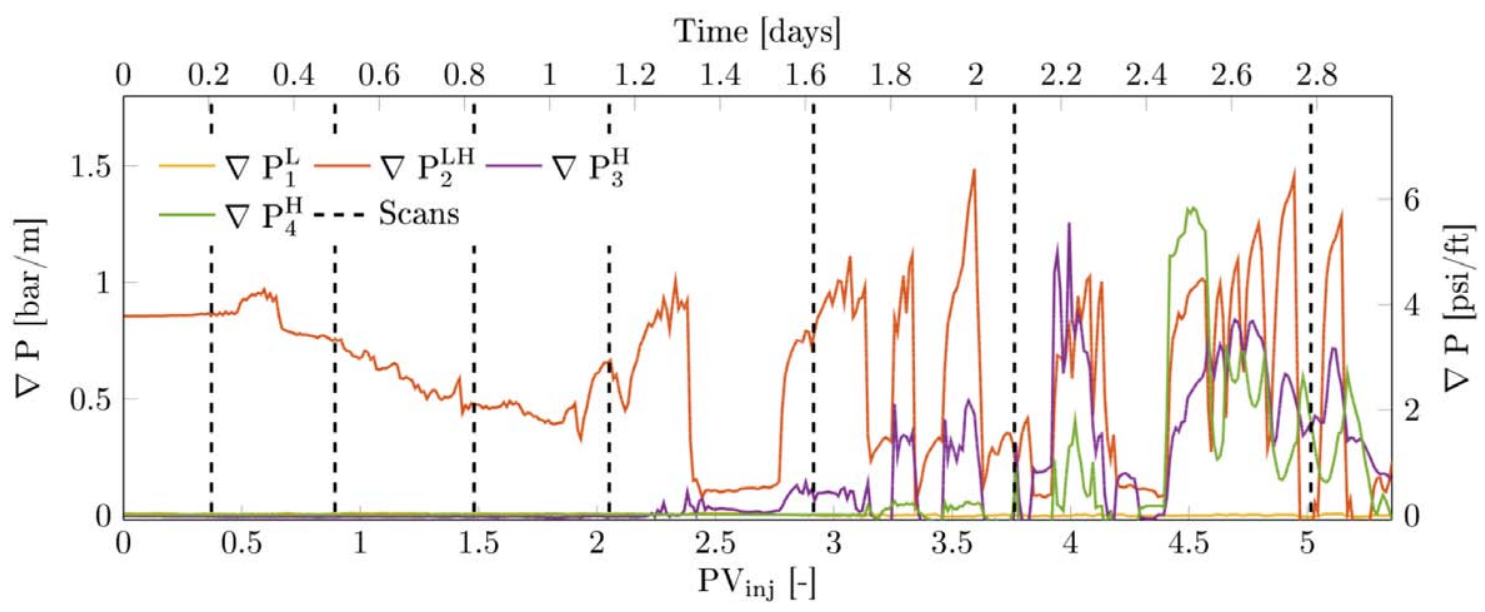

Figure 11-Pressure gradient across variations sections of core sample 3 during foam-generation experiment. Dashed lines indicate times at which CT scans were taken to generate saturation maps across the length of the core. Superscripts $L$ and $H$ denote the lowand high-permeability sections, respectively. 
CT images in Figure 12 also indicate that, as with sample 2, there may be a region of high permeability along the edges of the core due to imperfect sintering of the glass grains with the tube wall. Gas prefers to flow along the edges of the core and foam generation also begins at the outer boundary of the cylindrical porous medium, shown clearly in the scan taken at 2.1 PVI (Figure 12). Images at 2.9 and 3.8 PVI show that the foam front propagates first along the edges, forcing gas to flow through the center of the porous medium, resulting in subsequent propagation of foam through the center of the core.

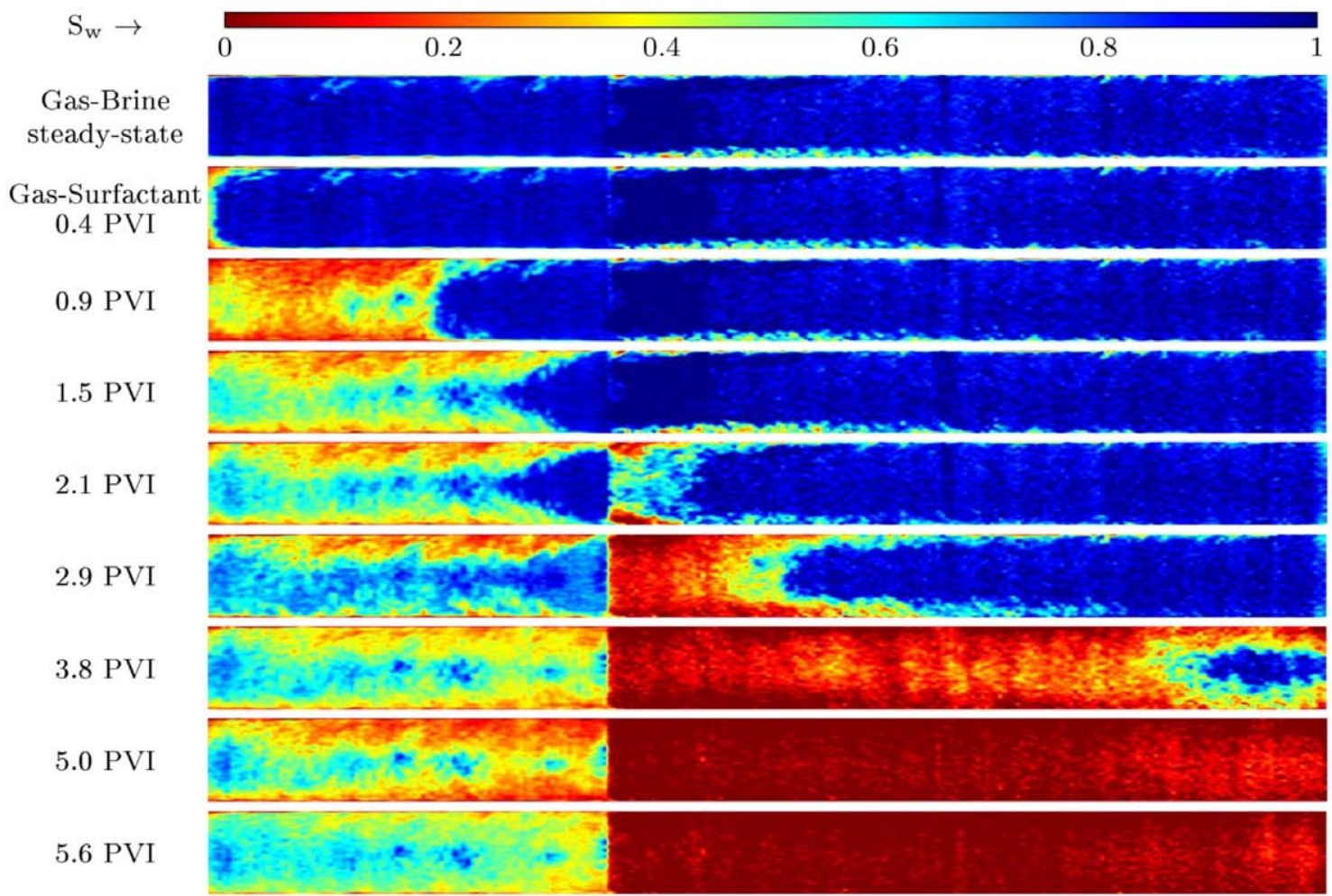

Figure 12-Liquid saturation profile in a vertical cross-section through the center of core sample 3 computed using $X$ ray $\mathrm{CT}$ imaging during the course of a foam-generation experiment. Color bar represents a liquid saturation range from 0 to 1. Blue represents a high liquid-phase saturation whereas red represents a high gas-phase saturation, here interpreted as the CT response to the saturation change caused by foam. Top-most saturation map comes from the CT scan taken during gas-brine injection and images thereafter are from scans taken after surfactant solution was introduced in the core.

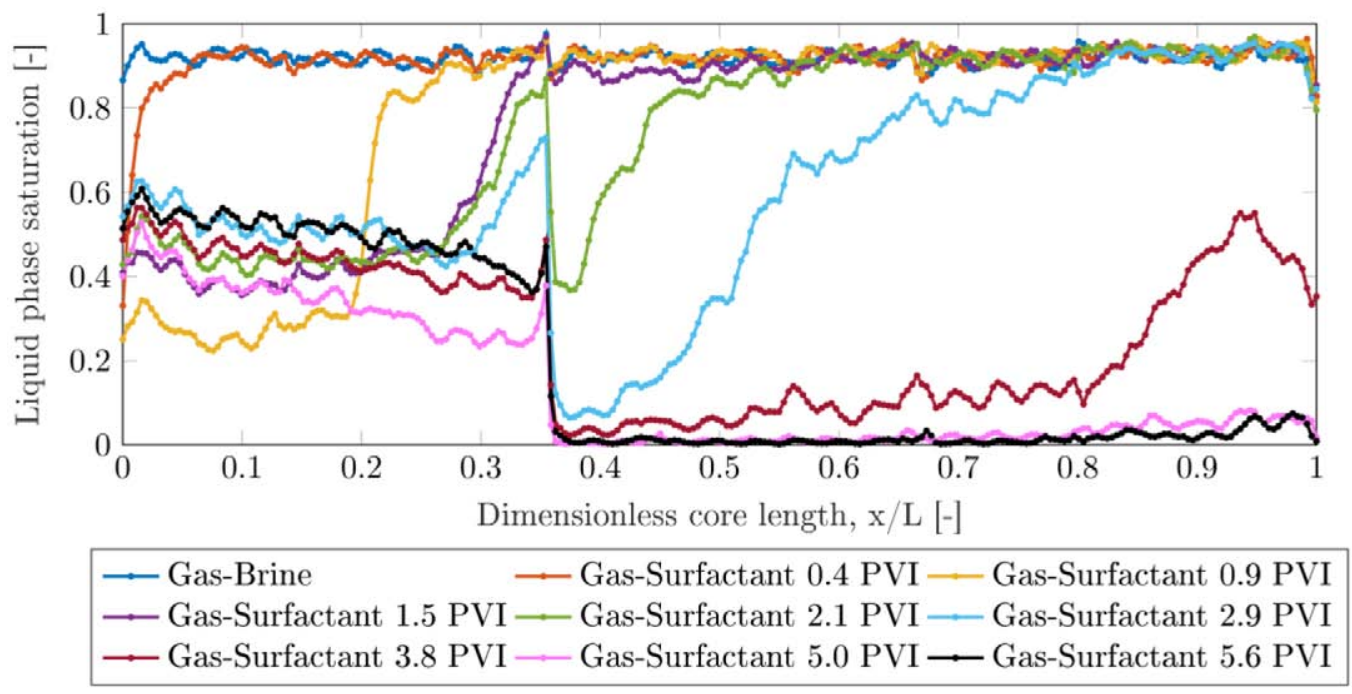

Figure 13-Average liquid saturation versus dimensionless core position during a foam-generation experiment in core sample 3. 


\section{Sample 4}

Core sample 4 had the highest permeability contrast among the tested cores. We observe that strong foam is created across the permeability transition and it propagates through the high-permeability zone to the end of the core. While the measured pressure gradient across the inlet section, as shown in Figure 14, shows no appreciable increase indicating absence of strong foam, the saturation profile obtained through CT scans, as shown in Figure 15, shows that the gas saturation is fairly high in the low-permeability zone, towards the end of the experiment. Gas saturation is almost $70 \%$ in the lowpermeability zone upstream of the transition as seen in the saturation profiles computed at 6.9 PVI and thereafter. This rise in gas saturation, however, is witnessed only after foam generation across the transition has been observed. In the high-permeability section, CT images show a gas saturation of almost 100\% towards the end of the experiment, indicating the presence of strong foam with a significant reduction in gas mobility, in terms of pressure gradient, as discussed in the next section.

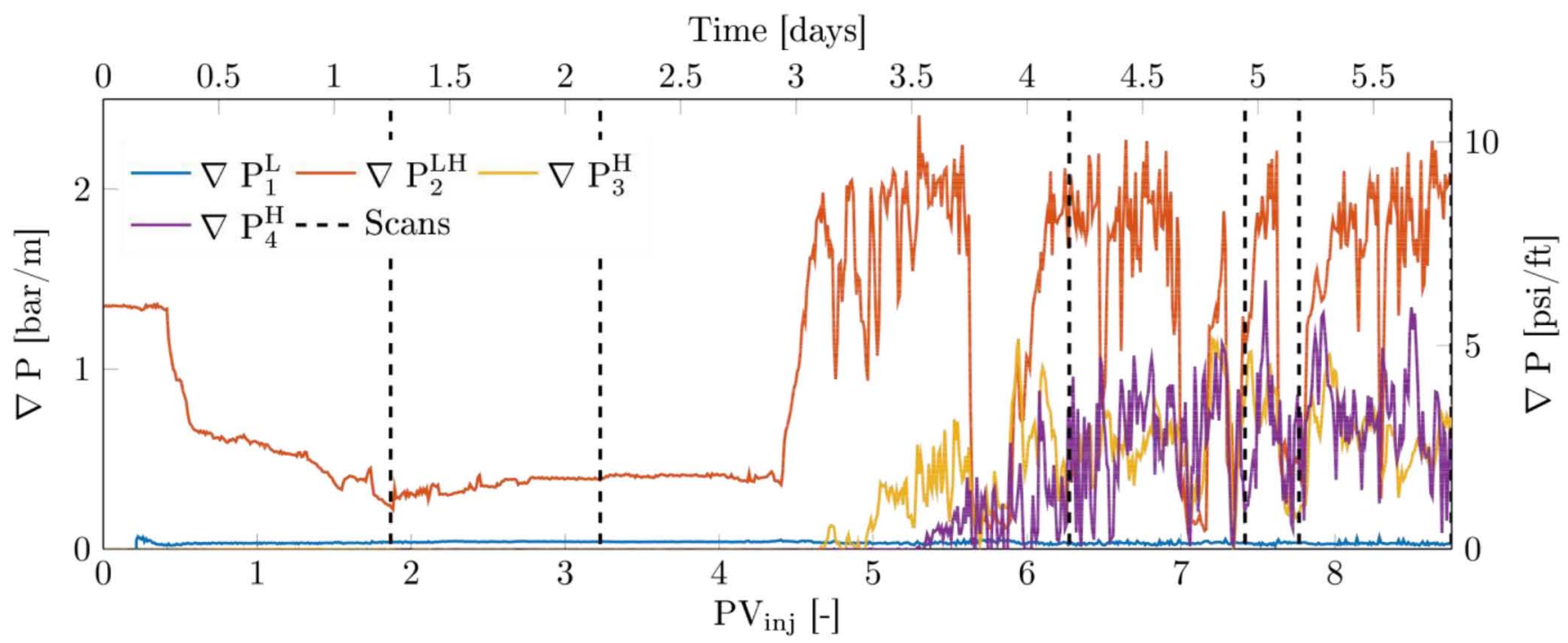

Figure 14-Pressure gradient across variations sections of core sample 4 during foam-generation experiment. Dashed lines indicate times at which CT scans were taken to generate saturation maps across the length of the core. Superscripts $L$ and $H$ denote the lowand high-permeability sections, respectively.

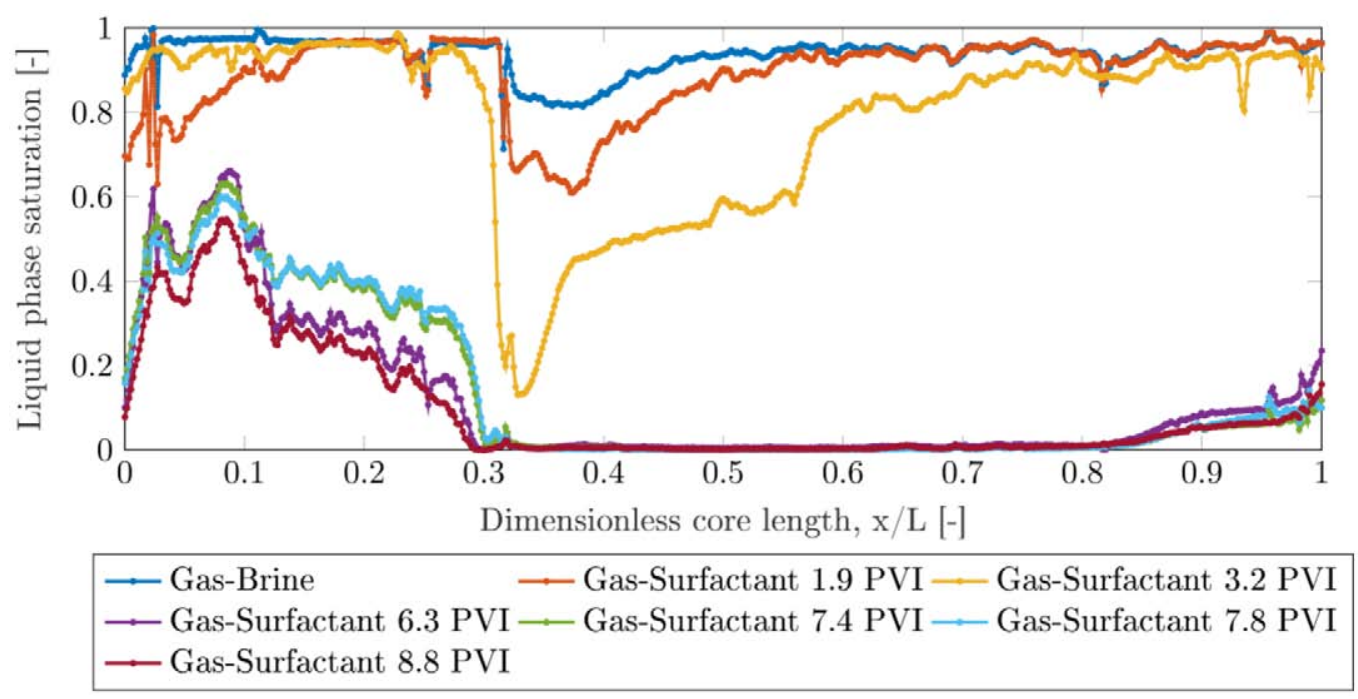

Figure 15-Average liquid saturation versus dimensionless core position during a foam generation experiment in core sample 4 


\section{Discussion}

In the experiments reported, we show evidence of foam generation across a sharp permeability rise during simultaneous flow of gas and surfactant solution. We observe foam generation across a permeability contrast of slightly less than $4: 1$ at a gas fractional flow of $80 \%$, in accordance with theoretical predictions. While in some of our experiments we see indications of foam generation in terms of high gas saturation in the lowpermeability section itself, this foam may be classified as "weak-foam" or what Friedmann et al. (1991) call a "leave-behind foam", as gas mobility remains high (in terms of measured pressure gradient) in this section throughout the course of the experiment.

Foam generation does result in a significant reduction in gas mobility in the high-permeability zone. We quantify this reduction in mobility in terms of apparent foam viscosity. Assuming a steady-state average pressure drop across the high-permeability zone, we use the pressure gradient across the entire zone and compute apparent viscosity as:

$$
\mu_{\text {app }}=\frac{\mathrm{k}^{\mathrm{H}} \nabla \mathrm{P}}{\mathrm{u}_{1}+\mathrm{u}_{\mathrm{g}}}
$$

where $\mathrm{k}^{\mathrm{H}}$ is the measured permeability of the high-permeability zone, $\nabla \mathrm{P}$ is the steady-state pressure gradient across the entire high-permeability zone measured after foam has propagated to the end of the core, and $u_{1}$ and $\mathrm{u}_{\mathrm{g}}$ are the superficial velocities of the liquid and gas phases, respectively. The apparent viscosity was averaged over quarter-day periods and plotted against total injected pore volumes in Figure 16. The error bar in each direction represents one standard deviation of the viscosities computed from all the recorded pressure gradients within the six-hour measurement window.

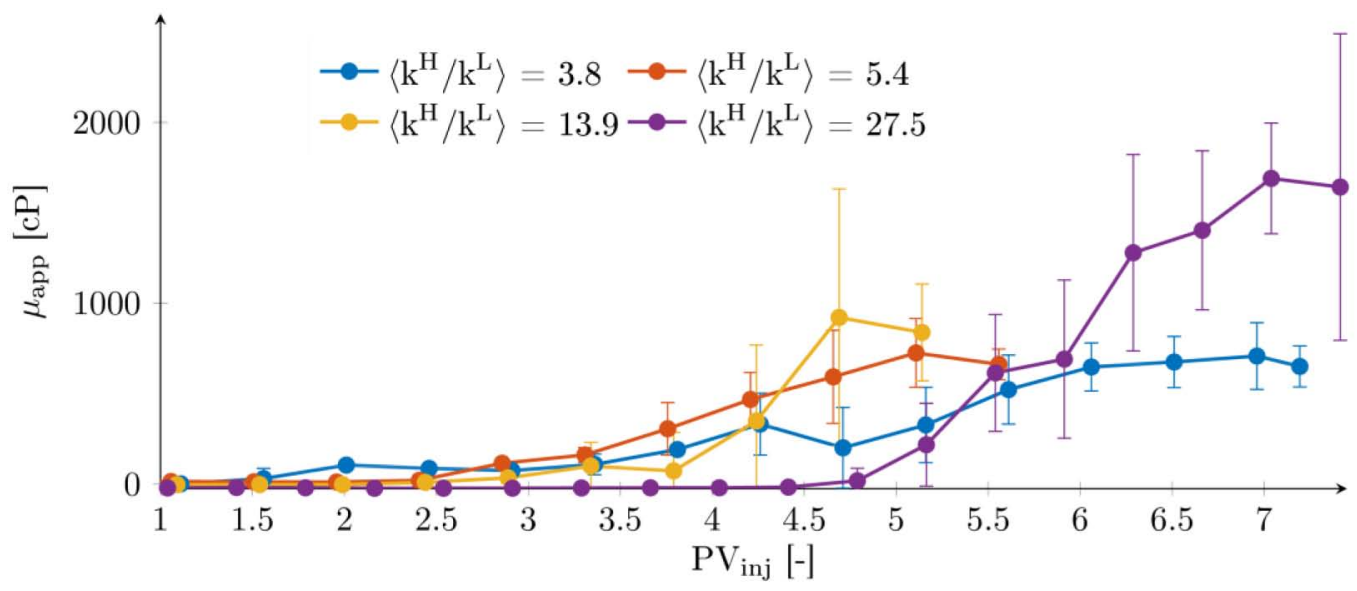

Figure 16-Apparent viscosity of foam in the high-permeability region of each core, generated during flow across the abrupt permeability increase, plotted against the total pore volumes of injection.

The average apparent viscosity of foam, generated across a sharp permeability increase, ranges from about $600 \mathrm{cP}$ to $1800 \mathrm{cP}$. Evidently, the greater the permeability contrast in the core, the larger the apparent viscosity of the generated foam. Additionally, we observe that at a greater permeability contrast, it takes longer to observe foam in the high-permeability section, especially in the core with the greatest permeability contrast, shown by the purple line in the above figure.

The measured pressure gradient in all our experiments exhibits large fluctuations. At a higher permeability contrast, the magnitude of these fluctuations is higher, as can be seen by the error bars in Figure 16. These fluctuations occur because lamellae are not steadily generated across the face of the heterogeneity (Falls et al., 1988). Foam generation across the sharp increase in permeability is intermittent. This can be explained as follows: foam generation causes a reduction in gas mobility, which in turn causes 
the flow to become drier; capillary pressure then rises above the critical capillary pressure for snap-off and foam generation ceases. This is more frequent at larger permeability contrasts. This coincides with our observations at the outlet of the core. We saw distinct periods of strong foam coming out of the core, followed by relatively weaker foam with a large bubble size, followed by periods of gas production only. The nature of produced fluids may also be affected by the capillary endeffect at the outlet. In a separate set of experiments, we have observed that the intermittency of foam generation and subsequent mobilization is less frequent at a higher liquid fractional flow and a higher flow rate.

\section{Conclusions}

Simultaneous flow of gas and surfactant solution across a sharp permeability jump results in foam generation by snap-off, provided that the liquid volume fraction is high enough and permeability contrast are sufficient. Foam can be generated by this phenomenon at extremely low superficial velocities, close to and even lower than conventional field velocities encountered far from wells. This coincides with theory, which suggests that this mechanism is independent of pressure gradient. CT images clearly indicate that foam is generated at the permeability change and it propagates downstream towards the outlet of the core.

At the low flow rates used in these experiments, no effective foam was generated in the homogeneous core upstream of the permeability transition. Events of foam generation and subsequent mobilization are associated with sharp fluctuations in the pressure gradient across the permeability jump. Foam generation and propagation is not immediate as the surfactant front reaches the permeability jump. Instead, foam generates, propagates and mobilizes intermittently from the permeability transition. The intermittency appears to be greater for greater permeability contrasts.

Foam generation during flow across abrupt permeability changes could significantly reduce gravity segregation, by reducing gas mobility (Jenkins, 1984; Stone, 1982). Foam generation can also occur during segregated flow across a permeability boundary. In such a case, foam is generated in the gas-rich layer and the subsequent reduction in gas mobility diverts gas, increasing sweep in the low-permeability layer.

\section{Acknowledgements}

This paper is a result of the collaboration between Delft University of Technology and Universiti Teknologi Petronas. We thank Petronas and Shell for funding this research. We gratefully acknowledge Michiel Slob, Ellen Meijvogel-de Koning, Marc Friebel and Karel Heller for their technical support at the Laboratory of Geoscience and Engineering of Delft University of Technology.

\section{References}

Ashoori, E., Marchesin, D., \& Rossen, W. R. 2012. Multiple Foam States and Long-Distance Foam Propagation in Porous Media. SPE Journal 17 (4): 1231-1245. SPE-15024-PA. https://doi.org/10.2118/154024-PA

Bernard, G. G., \& Holm, L. W. 1964. Effect of Foam on Permeability of Porous Media to Gas. SPE Journal 4 (3): 267-274. SPE-983-PA. https://doi.org/10.2118/983-PA

Falls, A. H., Hirasaki, G. J., Patzek, T. W., Gauglitz, D. A., Miller, D. D., \& Ratulowski, T. (1988). Development of a Mechanistic Foam Simulator: The Population Balance and Generation by Snap-Off. SPE Reservoir Engineering 3 (3): 884-892. SPE-14961-PA. https://doi.org/10.2118/14961-PA

Farajzadeh, R., Krastev, R., \& Zitha, P. L. J. 2008. Foam films stabilized with alpha olefin sulfonate (AOS). Colloids and Surfaces A: Physicochemical and Engineering Aspects 324 (1-3): 35-40. https://doi.org/10.1016/ J.COLSURFA.2008.03.024

Friedmann, F., Chen, W. H., \& Gauglitz, P. A. 1991. Experimental and Simulation Study of High-Temperature Foam Displacement in Porous Media. SPE Reservoir Engineering 6 (1): 37-45. SPE-17357-PA. https:// doi.org/10.2118/17357-PA

Friedmann, F., Smith, M. E., Guice, W. R., Gump, J. M., \& Nelson, D. G. 1994. Steam-Foam Mechanistic Field Trial in the Midway-Sunset Field. SPE Reservoir Engineering 9 (4): 297-304. SPE-21780-PA. https://doi.org/10.2118/21780-PA

Gauglitz, P. A., Friedmann, F., Kam, S. I., \& Rossen, W. R. 2002. Foam generation in homogeneous porous media. Chemical Engineering Science 57 (19): 4037-4052. https://doi.org/10.1016/S0009-2509(02)00340-8 
Hartkamp-Bakker, C. A. 1993. Permeability heterogeneity in cross-bedded sandstones: Impact on water/oil displacement in fluvial reservoirs. PhD Dissertation, Delft University of Technology, The Netherlands.

Hirasaki, G. J.; Jackson, R. E.; Jin, M.; Lawson, J. B.; Londergan, J.; Meinardus, H.; Miller, C. A.; Pope, G. A.; Szafranski, R.; Tanzil, D. 2000. Field Demonstration of the Surfactant/Foam Process for Remediation of a Heterogeneous Aquifer Contaminated with DNAPL. In NAPL Removal: Surfactants, Foams, and Microemulsions, S. Fiorenza, C. A. Miller, C. L. Oubre, \& C. H. Ward (Eds.), 3-163. Boca Raton: CRC Press.

Huh, D. G., \& Handy, L. L. 1989. Comparison of Steady and Unsteady-State Flow of Gas and Foaming Solution in Porous Media. SPE Reservoir Engineering 4 (1): 77-84. SPE-15078-PA. https://doi.org/10.2118/15078-PA

Jenkins, M. K. 1984. An Analytical Model for Water/Gas Miscible Displacements. Presented at the SPE Enhanced Oil Recovery Symposium, Tulsa, Oklahoma, 15-18 April. SPE-12632-MS. https://doi.org/10.2118/12632-MS

Kahrobaei, S., Li, K., Vincent-Bonnieu, S., \& Farajzadeh, R. 2017. Effects of compositional variations on $\mathrm{CO}_{2}$ foam under miscible conditions. AIChE Journal 4 (2): 758-764. https://doi.org/10.1002/aic.15938

Kam, S. I. 2008. Improved mechanistic foam simulation with foam catastrophe theory. Colloids and Surfaces A: Physicochemical and Engineering Aspects 318 (1-3): 62-77. https://doi.org/10.1016/j.colsurfa.2007.12.017

Kovscek, A. R., \& Radke, C. J. 1994. Fundamentals of Foam Transport in Porous Media. In Foams: Fundamentals and Applications in the Petroleum Industry, L. L. Schramm (Ed.), Chap. 3 115-163. Washington, DC: American Chemical Society.

Li, Q., \& Rossen, W. R. 2005. Injection Strategies for Foam Generation in Homogeneous and Layered Porous Media. Presented at the SPE Annual Technical Conference and Exhibition, Dallas, Texas, 9-12 October. SPE-96116-MS. https://doi.org/10.2118/96116-MS

McCool, C. S., Parmeswar, R., \& Willhite, G. P. 1983. Interpretation of Differential Pressure in Laboratory Surfactant/ Polymer Displacements. SPE Journal 23 (5): 791-803. https://doi.org/10.2118/10713-PA

Mees, F., Swennen, R., Geet, M. Van., \& Jacobs, P. 2003. Applications of X-ray computed tomography in the geosciences. Geological Society of London, Special Publications 215 (1): 1-6. https://doi.org/10.1144/GSL.SP.2003.21

Ransohoff, T. C., \& Radke, C. J. 1988. Mechanisms of Foam Generation in Glass-Bead Packs. SPE Reservoir Engineering 3 (2): 573-585. https://doi.org/10.2118/15441-PA

Reineck, H. E., \& Singh, J. B. (1980). Depositional Sedimentary Environments, second, revised and updated edition. Springer Berlin Heidelberg. https://doi.org/10.1007/978-3-642-81498-3

Rossen, W. R. 1996. Foams in enhanced oil recovery. In Foams: Theory, Measurements and Applications, R. K. Prud'homme \& S. A. Khan (Eds.), Chap. 11, 413-464. New York: Marcel Dekker.

Rossen, W. R. 1999. Foam Generation at Layer Boundaries in Porous Media. SPE Journal 4 (4): 409-412. SPE-59395PA. https://doi.org/10.2118/59395-PA

Rossen, W. R., \& Gauglitz, P. A. 1990. Percolation theory of creation and mobilization of foams in porous media. AIChE Journal 36 (8): 1176-1188. https://doi.org/10.1002/aic.690360807

Stone, H. L. 1982. Vertical, Conformance In An Alternating Water-Miscible Gas Flood. Presented at the SPE Annual Technical Conference and Exhibition, New Orleans, Louisiana, 26-29 September. SPE-11130-MS. https:// doi.org/10.2118/11130-MS

Tanzil, D., Hirasaki, G. J., \& Miller, C. A. 2002. Mobility of Foam in Heterogeneous Media: Flow Parallel and Perpendicular to Stratification. SPE Journal 7 (2): 203-212. SPE-78601-PA. https://doi.org/10.2118/78601-PA

Tanzil, D., Hirasaki, G. J., \& Miller, C. A. 2002. Conditions for Foam Generation in Homogeneous Porous Media. Presented at the SPE Improved Oil Recovery Symposium, Tulsa, Oklahoma, 13-17 April. SPE-75176-MS. https:// doi.org/10.2523/75176-MS

van Lingen, P. P. 1998. Quantification and Reduction of Capillary Entrapment in Cross-Laminated Oil Reservoirs. PhD Dissertation, Delft University of Technology, The Netherlands.

Yortsos, Y. C., \& Chang, J. 1990. Capillary effects in steady-state flow in heterogeneous cores. Transport in Porous Media 5 (4): 399-420. https://doi.org/10.1007/BF01141993 